\title{
Development of Subretinal Venous Sphincters in Spontaneously Hypertensive Rats (SHR)
}

\author{
S. Aharinejad*, U. Firbas \\ *Laboratory for Cardiovascular Research, Department of Anatomy, University of Vienna, \\ Waehringerstr. 13, A-1090 Vienna, Austria
}

Venous sphincters are tufts of smooth muscle that have been first described in the pancreatic and pulmonary microvascular bed of rats [1,2]. Venous sphincters are capable of contraction and depending on their tone, they can add a substantial resistance to the vascular pressure [1,2]. SHR are a strain bred of Wistar Kyoto (WKY) rats that spontaneously develop systemic hypertension [3]. In this strain, the pulmonary venous sphincters can contract deeply and narrow the luminal diameter of veins up to 50\% [4]. Existing reports indicate that SHR show hypertensive ocular fundus changes [5]. Other reports suggest that with ongoing systemic hypertension in SHR retinal vessels become tortuous, narrowed, and show localized constrictions [6]. However, it remains unknown whether venous sphincters are present in the ocular vasculature of SHR. We hypothesized that SHR might develop venous sphincters in their eyes and that the depth of sphincters' contraction might correlate with systemic blood pressure.

To examine our hypothesis, we used 16-18 week-old male SHR and age-matched WKY rats (Charles River, Germany). SHRs were untreated or treated with carvedilol, a combined $\alpha$ and $\beta$ receptor antagonist for 4 weeks. The systemic blood pressure was measured via a catheter in the abdominal aorta and recorded online using a telemetry system (Data Sciences International, Rochester, MN). The ocular vasculature was cast using Mercox CL-2B (Ladd Research, Williston, VT). For histology, the eyes were perfusion-fixed with paraformaldehyde. Cast specimens were prepared for scanning electron microscopy (SEM) as described earlier [1,4]. Histological specimens were sectioned serially and stained with $\mathrm{H}$ \& E.

In WKY rats the mean systemic blood pressure was $96 \pm 7$; in untreated SHR it was $148 \pm 15$; in carvedilol-treated SHR it was $131 \pm 10$ ( $P<0.001$ vs. untreated). SEM of the posterior eye pole in untreated SHR showed multiple focal constrictions on the surface of cast veins, narrowing the luminal diameter up to $40 \%$ (FIG. 1). In tissue sections, muscular tufts in the venous walls were readily identified (FIG. 2), corresponding to the venous sphincters [4]. These venous sphincters were located in the subretinal vascular network. In carvedilol-treated SHR only shallow constrictions were found on the surface of subretinal venous casts. The luminal surfaces of corresponding veins in WKY rats were smoothly outlined; venous sphincters were not found. This study suggests that venous sphincters are related and develop reactive to elevated systemic blood pressure in the ocular vasculature of SHRs. Venous sphincters might be of significance in regulating blood flow in the ocular vascular system.

\section{References}

[1] S. Aharinejad et al., Scanning 12 (1990) 280.

[2] D.E. Schraufnagel et al., Am. Rev. Respir. Dis. 141 (1990) 721. 
[3] K. Okamoto et al., Clin. Sci. Mol. Med. 45 (Suppl. 1) (1973) 11.

[4] S. Aharinejad et al., Am. J. Pathol. 148 (1996) 281.

[5] Y. Hamada et al., Clin. Exp. Pharmacol. Physiol. 22 (Suppl.) (1995) S132.

[6] I.A. Bhutto, T. Amemiya, Ophthalmic Res. 29 (1997) 12.
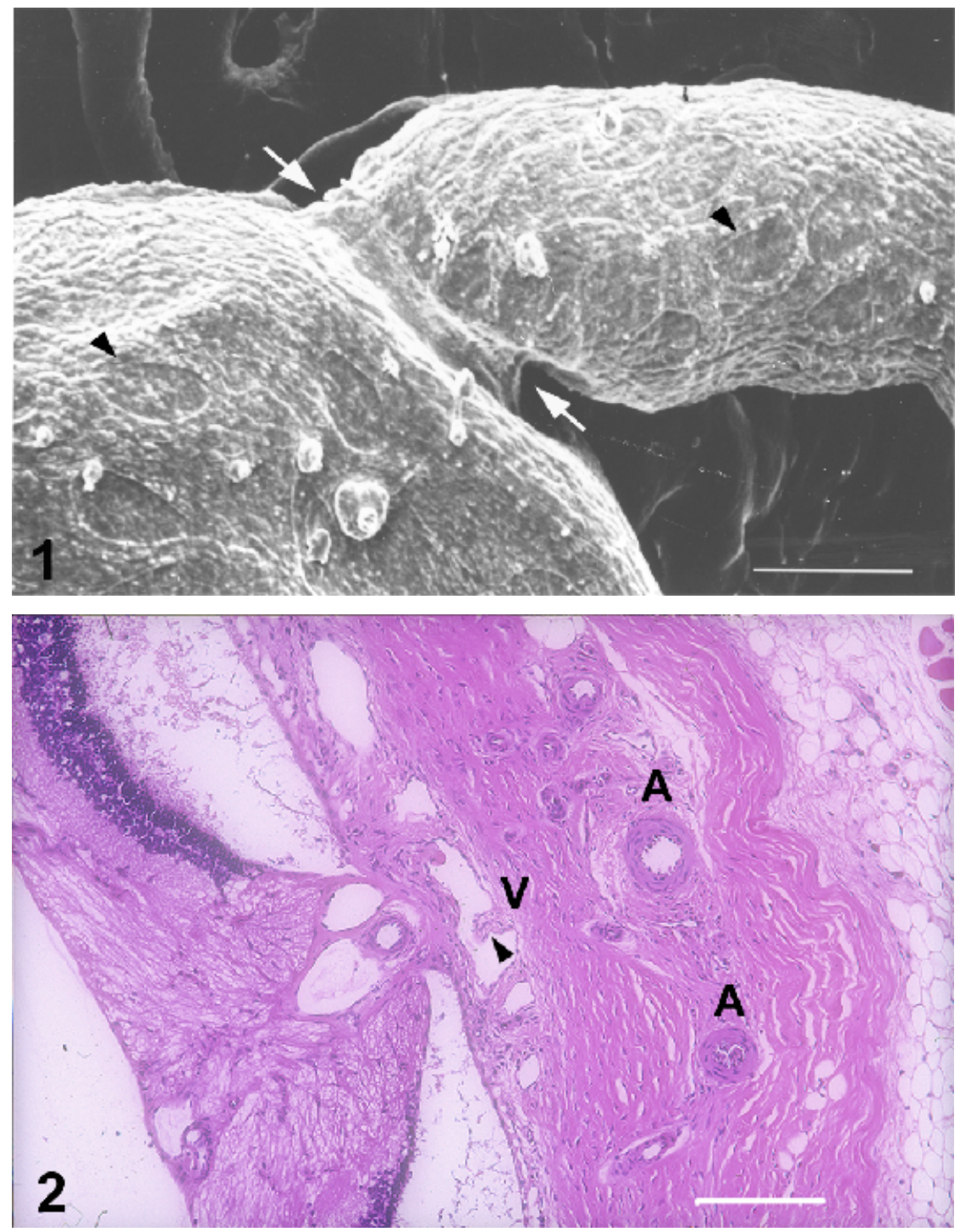

FIG. 1. SEM of a cast vein in the subretinal vascular network of an untreated SHR. White arrows mark a deeply contracted venous sphincter. Arrowheads mark characteristic endothelial cell nuclear imprints. $\mathrm{Bar}=25 \mu \mathrm{m}$.

FIG. 2. Light microscopy image of the ocular posterior pole of an untreated SHR. A smooth muscle sphincter (arrow head) bulges into the lumen of a longitudinally sectioned vein (V) in the subretinal vascular network. Two arteries (A) are seen close to the vein; note the hypertrophy of the media in the lower artery. Bar $=100 \mu \mathrm{m}$. 